\title{
Pulsations of the Low-Mass ZZ Ceti Star HS 1824+6000
}

\author{
Justin D. R. STEINFADT \\ Department of Physics, Broida Hall, University of California, Santa Barbara, CA; jdrs@physics.ucsb.edu \\ LARS BILDSTEN \\ Kavli Institute for Theoretical Physics and Department of Physics, Kohn Hall, University of California, Santa Barbara, CA; bildsten@kitp.ucsb.edu \\ AND \\ ERAN O. OFEK AND SHRi R. KULKARNI \\ Division of Physics, Mathematics and Astronomy, California Institute of Technology, Pasadena, CA \\ Received 2008 July 22; accepted 2008 August 27; published 2008 October 7
}

\begin{abstract}
Measuring $g$-mode pulsations of isolated white dwarfs can reveal their interior properties to high precision. With a spectroscopic mass of $\approx 0.51 M_{\odot}(\log g=7.82)$, the DAV white dwarf HS $1824+6000$ is near the transition between carbon/oxygen-core and helium-core white dwarfs, motivating our photometric search for additional pulsations from the Palomar 60-inch telescope. We confirmed (with much greater precision) the three frequencies: $2.751190 \pm 0.000010 \mathrm{mHz}(363.479 \mathrm{sec}), 3.116709 \pm 0.000006 \mathrm{mHz}(320.851 \mathrm{sec}), 3.495113 \pm$ $0.000009 \mathrm{mHz}(286.114 \mathrm{sec})$, previously found by B. Voss and collaborators, and found an additional pulsation at $4.443120 \pm 0.000012 \mathrm{mHz}(225.067 \mathrm{sec})$. These observed frequencies are similar to those found in other ZZ Ceti white dwarfs of comparable mass (e.g. $\log g<8$ ). We hope that future observations of much lower-mass ZZ Ceti stars $\left(<0.4 M_{\odot}\right)$ will reveal pulsational differences attributable to a hydrogen covered helium core.
\end{abstract}

\section{INTRODUCTION}

The hydrogen-line ZZ Ceti variable (DAV) white dwarfs (WDs) occupy a discrete strip in the $T_{\text {eff }}-\log g$ plane known as the ZZ Ceti instability strip. Many groups have assessed the location of this instability strip both empirically (Wesemael et al. 1991; Mukadam et al. 2004b; Gianninas et al. 2005; Castanheira et al. 2007) and theoretically (Brassard \& Fontaine 1997; Wu \& Goldreich 1999; Fontaine et al. 2003), and despite minor discrepancies it spans $11,000 \lesssim T_{\text {eff }} \lesssim 12,250 \mathrm{~K}$ for $\log g \approx 8.0$. Fontaine et al. (1982) suggested that the instability strip is pure, meaning that all WDs within the strip are variable. However, Mukadam et al. (2005) found numerous objects from the SDSS with associated low signal-to-noise spectra that were tenuously identified to be in the strip but did not vary to their observed detection limits. Recent observations (Castanheira et al. 2007) have found some of these to be low amplitude pulsators. If the instability strip is pure, then it strongly implies that ZZ Ceti stars are a phase of evolution through which all DA WDs must evolve as they cool.

White dwarfs less massive than $\approx 0.45-0.47 M_{\odot}(\log g \approx$ 7.67 at $T_{\text {eff }} \approx 11,500 \mathrm{~K}$ ) (D'Cruz et al. 1996; Dominguez et al. 1999; Pietrinferni et al. 2004; VandenBerg et al. 2006; Panei et al. 2007) do not undergo a He-core flash in the course of their evolution and therefore are left with a He core. Two modes of evolution can truncate the red giant branch evolution and prevent the He-core flash: mass loss due to winds and mass loss due to binary interaction. In systems of high metallicity, mass loss due to stellar winds on the red giant branch can be significant enough to lose the $\mathrm{H}$ envelope prior to the core flash (D'Cruz et al. 1996; Hansen 2005; Kilic et al. 2007b). Binary interaction through a common envelope also leads to significant mass loss (Iben \& Livio 1993; Marsh et al. 1995). Helium is thus the expected core composition for WDs below $\approx 0.45-0.47 M_{\odot}$. However, little direct evidence exists of the He core. Possible evidence would be the apparent overbrightness of old WDs (Hansen 2005) in the star cluster NGC 6791 (Bedin et al. 2005). Though uncertainties remain (Deloye \& Bildsten 2002; Bedin et al. 2008a, 2008b), recent detection of low $\log g$ young WDs (Kalirai et al. 2007) makes it plausible for many of the old WDs to be He core. A detailed asteroseismological study of these low-mass WDs could provide convincing evidence for the core composition. Observation and analysis of a full spectrum of the pulsation modes in a WD can produce a wealth of information about the interior structure of the WD. The mean period spacing of the modes, the rate of change in a mode's period over time, and multiplet splitting of individual modes can provide information on the total mass, spin rate, magnetic field strength, mass of $\mathrm{H}$ envelope, and core composition of the WD (Córsico \& Benvenuto 2002; Castanheira \& Kepler 2008). This has already been theoretically applied to distinguish between $\mathrm{C} / \mathrm{O}-$ and $\mathrm{O} / \mathrm{Ne}$-core WDs by Córsico et al. (2004). The measured change in an 


\section{STEINFADT ET AL.}

observed mode period in G117-B15 has also been used to constrain significantly the $\mathrm{C} / \mathrm{O}$-core composition of this object (Kepler et al. 1991, 1995, 2000, 2005a).

We plot a version of the empirical instability strip in Figure 1. Included are not-observed-to-vary (NOV) systems and pulsating ZZ Ceti stars from the observations of Bergeron et al. (2004) and Gianninas et al. (2005, 2007). Also included are low-mass WDs from Kilic et al. (2007a). There is a notable absence of low-mass $(\log g \lesssim 7.67)$ WDs within the instability strip. There are a few possible ZZ Ceti stars of this low mass that are not plotted due to the absence of spectroscopically determined $\log g$ and $T_{\text {eff }}$ measurements (Voss et al. 2007). Also shown are the He WD cooling tracks of two models for $\approx 0.19,0.24,0.40$, and $0.45 M_{\odot}$ WDs (Althaus et al. 2001; Panei et al. 2007). The difference between these two models at low mass is due to the different $\mathrm{H}$ envelope masses. Althaus et al. (2001) used a $1 M_{\odot}$ main-sequence star and truncated its evolution up the red giant branch at various stages to produce He WDs of varying masses. Panei et al. (2007) used close binary evolution expectations for main-sequence stars of many masses to produce He WDs of varying masses. These different approaches cause the different remnant $\mathrm{H}$ envelope masses that yield a degeneracy in the He WD mass and its position in the
$T_{\text {eff }}-\log g$ plane. This degeneracy would be broken in the case of a ZZ Ceti He WD where the pulsation mode spectrum would reveal the $\mathrm{H}$ envelope mass.

HS 1824+6000 (hereafter HS 1824; see Table 1) was initially observed by Voss et al. (2006) to exhibit pulsations. Their photometrically determined $\log g$ and $T_{\text {eff }}$ placed its mass at $\approx 0.40 M_{\odot}$ using the tables of Althaus \& Benvenuto (1997). This mass was well within the theoretical expected mass range for He-core WDs making it an excellent object to compare and contrast its pulsation frequencies with other $\mathrm{C} / \mathrm{O}$-core and possible He-core DAVs. However, later spectroscopic measurement by Gianninas et al. (2007) determined its mass to be $\approx 0.51 M_{\odot}$, beyond the expected mass range for He-core WDs. In $\S 2$ we discuss our own observations and differential photometry of HS 1824. In $\S 3$ we apply a Lomb-Scargle Periodogram approach to a nonuniformly sampled time series in order to obtain the pulsation frequencies of HS 1824. In $\S 4$ we report the results of our observations and analysis.

In $\S 5$ we compare all observed $\mathrm{ZZ}$ Ceti periods with $\log g<8.0$. It is our hope this will yield a "zeroth-order" approach to He-core identification in much the same way $T_{\text {eff }}$ and $\log g$ measurements of field WDs identify likely ZZ Ceti stars. No singular distinction is currently present.

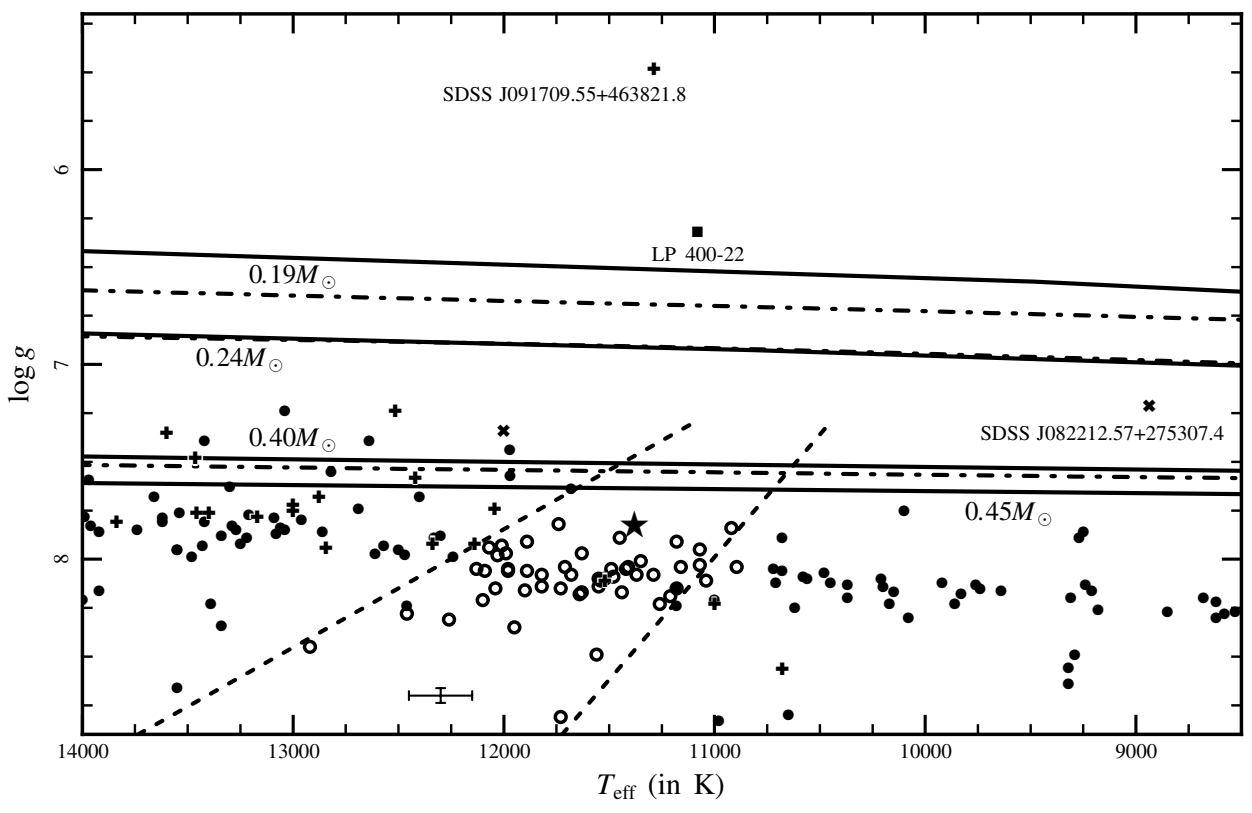

FIG. 1.-Empirical ZZ Ceti instability strip. The circles represent systems for which temporal observations have been performed. Filled circles indicate systems not observed to vary, while open circles indicate systems with observed periods. These data are from Bergeron et al. (2004) and Gianninas et al. (2005, 2007). The vertical crosses represent low-mass WDs selected from the SDSS with $\log g$ and $T_{\text {eff }}$ redetermined from MMT spectra from Kilic et al. (2007a). The diagonal crosses are from Kilic et al. (2007a), except that they are reanalysis of SDSS spectra and are merely candidate low-mass WDs until better spectra can be obtained. The square is LP 400 -22 (Kawka et al. 2006; Kilic et al. 2007a). The star is HS 1824+6000 (see Table 1). The error bar in the instability strip represents the typical error for measurements within the instability strip. The dashed lines are empirical fits to the instability strip as determined by Gianninas et al. (2007). The solid (Panei et al. 2007) and dash-dotted (Althaus et al. 2001) lines correspond to cooling tracks of He WDs of the labeled mass. The labeled masses correspond to the following model masses (in $M_{\odot}$ ) for Panei et al. (2007) and Althaus et al. (2001): 0.19 to 0.1869 and 0.196, 0.24 to 0.2495 and $0.242,0.40$ to 0.3986 and $0.406,0.45$ to 0.4481 (Panei et al. 2007 only). 
TABLE 1

PROPERTIES OF HS $1824+6000$

\begin{tabular}{|c|c|c|}
\hline & Voss et al. 2006 & Gianninas et al. 2007 \\
\hline$T_{\text {eff }}(\mathrm{K}) \quad \ldots \ldots \ldots \ldots$ & $11192 \pm 300^{\mathrm{a}}$ & $11380 \pm 140^{\mathrm{b}}$ \\
\hline $\log g(\operatorname{dex}) \ldots \ldots \ldots \ldots$ & $7.65 \pm 0.10^{\mathrm{a}}$ & $7.82 \pm 0.04^{\mathrm{b}}$ \\
\hline$m_{B}(\operatorname{mag}) \quad \ldots \ldots \ldots \ldots$ & 15.7 & 15.7 \\
\hline \multicolumn{3}{|c|}{ Observed Frequencies (in $\mathrm{mHz}$ ) } \\
\hline Voss et al. $2006 \ldots$.... & $\begin{array}{c}2.6 \pm 0.4,3.0 \pm 0.9,3.3 \\
\pm 0.4,3.4 \pm 0.8\end{array}$ & \\
\hline This Paper & $\begin{array}{r}2.751190 \pm 0.000010 \\
3.116709 \pm 0.000006 \\
3.495113 \pm 0.000009 \\
4.443120 \pm 0.000012\end{array}$ & \\
\hline
\end{tabular}

${ }^{\text {a }}$ Photometrically determined.

${ }^{\mathrm{b}}$ Spectroscopically determined.

\section{OBSERVATIONS}

We observed HS 1824 (see Table 1 for properties) on 11 nights from 2006 August to 2006 October using the robotically operated 60 -inch $(1.52-\mathrm{m})$ telescope at the Palomar Observatory (Cenko et al. 2006). All observations had $30 \mathrm{~s}$ exposures with dead times ranging 20-40 s. To reduce dead time, half the CCD was read out. The large variance in the dead time was due primarily to a technical problem within the automated observing software used to control the telescope. The observing durations varied from 1 to $3 \mathrm{hr}$. The primary $2048 \times 2048$ pixel, $11^{\prime} \times 11^{\prime}$, CCD for the robotic Palomar 60-inch was used in all observations with a Gunn $g$ filter. We chose the Gunn $g$ filter to optimize the ratio of pulsation count amplitude to total stellar counts. It is a known trend that this ratio is larger in bluer filters such as Gunn $g$ (Robinson et al. 1982, 1995). A clear filter would not be optimal as it increases the total stellar counts without a comparable increase in pulsation count amplitude, thus reducing this important ratio. Flat fielding, bias subtraction, and sky subtraction were performed within the data pipeline of the Palomar 60-inch Telescope Archive (Cenko et al. 2006). The sky subtraction was done as an inaccurate scalar value and for our purposes was added back into the data and recalculated using standard, more accurate IRAF $^{1}$ tools.

\subsection{Data Reduction}

We used the IRAF package VAPHOT (Deeg \& Doyle 2001) to dynamically determine optimum aperture sizes as a function of seeing for our photometry. Given a characteristic frame for each night, VAPHOT calculates an optimized aperture using a PSF for each star that maximizes the signal-to-noise ratio within the aperture. This optimized aperture is then found as

${ }^{1}$ IRAF (Image Reduction and Analysis Facility) is distributed by the National Optical Astronomy Observatory, which is operated by the Association of Universities for Research in Astronomy, Inc., under contract with the National Science Foundation. More information is available online at http://iraf.noao.edu. a function of the seeing. Then, for a time series of frames, VAPHOT calculates the seeing value for each individual frame and scales the optimized apertures accordingly. Finally, all aperture information is input into the standard IRAF task phot, which calculates counts within the optimized aperture along with background noise counts measured in an annulus just beyond the optimized aperture. For each night of observation, 21 comparison stars were selected ranging $g=12-16$ mag and along with the program star, counts and background information were extracted using the VAPHOT task. Additionally, the exposure start times for all frames of observation were converted to barycentric Julian dates.

The uncertainty of this photometry for each aperture is given by

$$
\sigma_{\mathrm{CCD}}^{2}=c+n_{\text {bins }}\left(1+\frac{n_{\text {bins }}}{n_{\text {sky }}}\right)\left(N_{S}+N_{R}^{2}+N_{D}\right)
$$

(Howell 2006), where $c$ is the number of integrated source counts in photons, $n_{\text {bins }}$ is the aperture area calculatedby VAPHOT in pixels, $n_{\text {sky }}$ is the area of the annulus used to calculate the background information in pixels, $N_{S}$ is the background counts per pixel, $N_{R}$ is the read noise of the CCD in counts per pixel, and $N_{D}$ is the dark current in counts per pixel. We do not include the digitization error as it is significantly less than our value for the gain. For our observations, $c$ was $\approx 4 \times$ $10^{4}$ counts for HS 1824 and $\approx 10^{4}-10^{6}$ counts for the comparison stars, while $N_{S} \approx 40-600$ counts, $N_{R}^{2} \approx 25-60$, and $N_{D}$ $\ll 1$ counts for $t_{\text {int }}=30 \mathrm{~s}$ integrations.

Additional uncertainty arises from atmospheric variability on spatial scales of the CCD field of view. Scintillation is a dimensionless measure of the flux variations of a source observed through a finite aperture (our telescope) due to fluctuations in the refractive index of the atmosphere caused by temperature changes. Young's formulation (Young 1967) of Reiger's theory of scintillation (Reiger 1963) gives $s_{\text {scint }}=S_{0} d^{-2 / 3} X^{3 / 2} e^{-h / h_{0}} \Delta f^{1 / 2}$, where $S_{0}=0.09$ is a constant (Young 1967), $d=152 \mathrm{~cm}$ is the mirror diameter, $X$ is the airmass, $h=1706 \mathrm{~m}$ is the Palomar Observatory altitude, $h_{0}=8000 \mathrm{~m}$ is a constant (Young 1967), and $\Delta f=1 / t_{\text {int }}$. The formal photometric error for each star in each frame is

$$
\sigma^{2}=\sigma_{\mathrm{CCD}}^{2}+s_{\mathrm{scint}}^{2} c^{2}
$$

which determines the count level, $c$, at which scintillation noise becomes comparable to Poisson noise. This occurs at $9 \times 10^{5}$ counts at airmass 1.15 and $2 \times 10^{5}$ counts at airmass 2.0. Compared to the total formal error given by Equation (2), scintillation accounts for $10 \%-40 \%$ of the error depending upon the airmass (higher airmass accounts for higher percentages). Therefore, we are mostly limited by Poisson counting statistics, but scintillation can become significant at higher airmass. 


\subsection{Differential Photometry}

Because the atmosphere is constantly changing, differential rather than absolute photometry was used in the construction of our light curves. We used an ensemble of comparison stars to reduce the noise level inherent in any single comparison star. We used the weighting scheme detailed in Sokoloski et al. (2001) inspired by Gilliland \& Brown (1988). For our target star we define

$$
\begin{gathered}
x(i)=A \frac{c_{p}(i)}{\sum_{m=1}^{K} w_{m} c_{m}(i)}, i=1, \ldots, N, \\
\sigma_{x}^{2}(i) \approx\left[\frac{\sigma_{p}(i)}{c_{p}(i)}\right]+\frac{\sum_{m=1}^{K}\left[w_{m} \sigma_{m}(i)\right]^{2}}{\left[\sum_{m=1}^{K} w_{m} c_{m}(i)\right]^{2}}, \\
A^{-1}=\frac{1}{N} \sum_{i=1}^{N} \frac{c_{p}(i)}{\sum_{n=1}^{K} w_{n} c_{n}(i)}, \\
w_{m}=\frac{\sum_{i=1}^{N} c_{m}(i)}{\sum_{i=1}^{N} \sigma_{m}^{2}(i)},
\end{gathered}
$$

where $x(i)$ is the count ratio for the $i$ th image, $c_{p}(i)$ and $\sigma_{p}(i)$ are the background-subtracted counts and uncertainty of the program star, $c_{m}(i)$ and $\sigma_{m}(i)$ are the background-subtracted counts and uncertainty for the $m$ th comparison star in the $i$ th image, $K$ is the number of comparison stars, and $N$ is the total number of frames in the light curve. The weights of the $m$ th comparison star, $w_{m}$, are the same for every image, while $A$ is a normalization factor that gives $x(i)$ meaning such that

$$
\Delta c_{p}(i)=\bar{c}_{p}[x(i)-1]
$$

where $\bar{c}_{p}$ is the mean background-subtracted counts of the program star and $\Delta c_{p}(i)$ is the difference in the total counts of the $i$ th frame compared to the mean counts of the program star for that night.

\section{LOMB-SCARGLE TIMING ANALYSIS}

The robotically controlled Palomar 60-inch presents a few challenges for time domain observations. First is the variable dead time of 20-40 s after a $30 \mathrm{~s}$ exposure. Second, the automated observing program sometimes places a higher priority on other targets, thus placing temporal gaps in our time series. While data gaps can be addressed in discrete Fourier analysis, large variations in timing present a much more difficult problem that we address via the Lomb-Scargle periodogram approach.

Scargle (1982) defines a periodogram as a function of the angular frequency $\omega$ (in $\operatorname{rads}^{-1}$ ) as follows:

$$
\begin{aligned}
& P_{x}(\omega)=\left(\frac{1}{2} \frac{\left\{\sum_{i=1}^{N} x\left(t_{i}\right) \cos \left[\omega\left(t_{i}-\tau\right)\right]\right\}^{2}}{\sum_{i=1}^{N} \cos ^{2}\left[\omega\left(t_{i}-\tau\right)\right]}\right. \\
&\left.+\frac{\left\{\sum_{i=1}^{N} x\left(t_{i}\right) \sin \left[\omega\left(t_{i}-\tau\right)\right]\right\}^{2}}{\sum_{i=1}^{N} \sin ^{2}\left[\omega\left(t_{i}-\tau\right)\right]}\right), \\
& \tan (2 \omega \tau)=\frac{\sum_{i=1}^{N} \sin \left(2 \omega t_{i}\right)}{\sum_{i=1}^{N} \cos \left(2 \omega t_{i}\right)}
\end{aligned}
$$

where $x\left(t_{i}\right)$ is $\Delta c_{p}(i)$ from our differential photometry $(\$ 2.2)$ and $t_{i}$ is the start time in seconds of the $i$ th frame. Further considerations by Scargle (1982) and Horne \& Baliunas (1986) showed that the probability distribution of power at frequency $\omega$ (with Gaussian white noise) is $\operatorname{Prob}[\mathrm{P}(\omega)>z]=e^{-z}$ when the periodogram is normalized as

$$
P(\omega)=P_{x}(\omega) / \sigma^{2}
$$

where $\sigma^{2}$ is the total measured variance of $x(t)$ over the entire time series. Horne \& Baliunas (1986) also showed that for data with periodic signals, the normalization factor remains the total variance of the raw data with the signal present.

The $\exp (-z)$ probability distribution quantifies the significance of any signal seen within the periodogram, allowing us to find the probability that the noise (presumed to be independent and normal) would, by itself, produce a power of $z$. This allows us to generate a false-alarm probability that states if we scan $M$ independent frequencies then the probability that the intrinsic noise produces a power greater than $z$ in any one of the frequency bins is

$$
\operatorname{Prob}(\text { Any Power }>z)=1-\left(1-e^{-z}\right)^{M} \text {. }
$$

A periodic signal is thus significant to $90 \%$ over all $M$ sampled frequencies if the false-alarm probability is $10 \%$. Because our noise is not exactly normally distributed due to the presence of unresolved pulsations that assure some correlation between data points, the precise significance may be slightly lower than this. However, this will not have any consequence for our results.

\section{FINAL LIGHT CURVES AND PERIODOGRAMS: RESULTS}

All 21 comparison stars would not produce the most stable comparison set. To determine the optimum ensemble of comparison stars for a given observing run, every comparison star was compared to all other comparison stars one at a time by calculating light curves ( $\$ 2.2$ ) and periodograms ( $\$ 3)$. Such an analysis reveals consistent frames where a comparison star has a count value much beyond the scatter of the normal light curve. In these cases, that frame and comparison star were analyzed using standard IRAF tasks to determine what caused the contamination (e.g., cosmic ray strike, drift into bad pixel due to 
poor guiding). Almost always, these comparison stars were then excluded from the optimum comparison star ensemble for that night only. The periodograms found those comparison stars with consistent frequency content due to possible intrinsic variability. These comparison stars were also excluded. Individual frames were excluded when the program star was contaminated by cosmic ray strikes, or the entire frame was affected by a high background level or poor seeing.

Comparison stars were also excluded when color-airmass effects could not be adequately removed via a detrending secondorder polynomial. This was done by comparing all comparison stars to HS 1824 individually and looking for high levels of noise in the lowest frequency domain of the periodogram. Detrending with a second-order polynomial is acceptable in our situation as the periods of pulsation are much shorter than the hours timescale it takes for changing color-airmass. The resulting optimum comparison star ensemble was then used to compute the HS 1824 differential light curve (§ 2.2), which was detrended through the second-order polynomial fitting, and the final light curve processed through the periodogram (§3).

Using Equation (7), we determined an observed power to be significant in any periodogram if the probability was greater than $90 \%$ (false-alarm probability less than $10 \%$ ) over all sampled frequencies. A summary of all significant frequencies is in Table 2, where the frequency uncertainty reported is the separation of the frequency bins in the periodogram. Figure 2 shows the differential light curves and periodograms for our four longest data sets. The data from the four longest observations allow us to construct a weighted average to arrive at $2.73 \pm 0.03 \mathrm{mHz}(366 \mathrm{~s}), 3.12 \pm 0.02 \mathrm{mHz}(321 \mathrm{~s}), 3.53 \pm$
$0.03 \mathrm{mHz}(283 \mathrm{~s})$, and $4.45 \pm 0.05 \mathrm{mHz}(225 \mathrm{~s})$. The 2.7 and $4.4 \mathrm{mHz}$ frequencies are confirmed in three nights, the $3.5 \mathrm{mHz}$ frequency in two nights, and the $3.1 \mathrm{mHz}$ frequency in nine nights. Excess power is often observed in these frequencies on other nights although not to the required significance level $(90 \%)$.

Our final analysis combined all 11 nights of data into one data set. Sky conditions were not the same for all nights, so the individually reduced data as described above was used and then combined. Barycentric Julian dates must be used in this analysis as changes in the Earth's orbital position in the solar system can account for as much as a $8 \mathrm{~s}$ per day change in light arrival time. This composite data set was spectrally analyzed using the Lomb-Scargle periodogram and the result is plotted in Figure 3.

All four detected frequencies are recovered to our $90 \%$ confidence; however, the imprint of our window function makes it difficult to determine any gains in precision over the individual nights. To address this concern we used a method of least-squares fitting of sinusoids at all four detected frequencies, allowing a single frequency to vary while fixing the remaining frequencies and minimizing $\chi_{\mathrm{fit}}^{2}$. The old detected frequency was then replaced with this more accurate frequency. This was done for all four frequencies and repeated recursively until all four frequencies no longer changed values significantly. This method gives us accurate determinations of the frequencies, amplitudes, and phases of the four detected frequencies. To determine the precision of these new measurements we used a more robust $\chi_{\text {fit }}^{2}$ minimization technique allowing all parameters to vary, now including the frequencies. The inherent nonlinearity

TABLE 2

Observation Dates and Pulsation Results FOR HS 1824+6000

\begin{tabular}{|c|c|c|c|c|c|c|c|}
\hline $\begin{array}{l}\text { Date (UT) } \\
\text { YYMMDD }\end{array}$ & $\begin{array}{l}2.7 \text { (Ampl.) } \\
\mathrm{mHz} \text { (mmag) }\end{array}$ & $\begin{array}{l}3.1 \text { (Ampl.) } \\
\text { mHz (mmag) }\end{array}$ & $\begin{array}{l}3.5 \text { (Ampl.) } \\
\mathrm{mHz} \text { (mmag) }\end{array}$ & $\begin{array}{l}4.4 \text { (Ampl.) } \\
\text { mHz (mmag) }\end{array}$ & $\begin{array}{l}\text { Obs. } \\
\text { Length }^{\mathrm{a}} \\
\mathrm{hr}\end{array}$ & $\begin{array}{c}\text { Number } \\
\text { of } \\
\text { Frames }^{\mathrm{a}}\end{array}$ & $\begin{array}{c}\text { Number of } \\
\text { Comp. } \\
\text { Stars }^{\mathrm{b}}\end{array}$ \\
\hline $060821 \ldots \ldots$ & $\ldots$ & $\ldots$ & $\ldots$ & $4.45 \pm 0.10(9.7)$ & 0.8 & 56 & 12 \\
\hline 060824 & $2.68 \pm 0.16(8.1)$ & $3.15 \pm 0.16(9.1)$ & $\ldots$ & $\ldots$ & 0.9 & 59 & 13 \\
\hline 060827 & $\ldots$ & $3.08 \pm 0.15(10.4)$ & $\ldots$ & $\ldots$ & 0.9 & 59 & 14 \\
\hline $060830 \quad \ldots$. & $\ldots$ & $3.14 \pm 0.13(6.2)$ & $\ldots$ & $4.44 \pm 0.13(5.9)$ & 1.1 & 69 & 11 \\
\hline $060903 \ldots \ldots$ & $\ldots$ & $\ldots$ & $\ldots$ & $\ldots$ & 1.0 & 68 & 11 \\
\hline $060906 \ldots$. & $\ldots$ & $3.13 \pm 0.14(7.8)$ & $\ldots$ & $\ldots$ & 1.0 & 69 & 14 \\
\hline $060909 \ldots \ldots$ & $\ldots$ & $3.15 \pm 0.12(11.8)$ & $\ldots$ & $\ldots$ & 1.2 & 66 & 10 \\
\hline $061009 \quad \ldots$. & $2.74 \pm 0.05(5.6)$ & $3.13 \pm 0.05(7.5)$ & $\ldots$ & $4.45 \pm 0.05(7.0)$ & 2.8 & 137 & 9 \\
\hline $061016 \ldots \ldots$ & $\ldots$ & $3.10 \pm 0.03(8.1)$ & $3.53 \pm 0.03(5.3)$ & $\ldots$ & 3.5 & 129 & 9 \\
\hline $061019 \ldots \ldots$ & $2.73 \pm 0.03(7.4)$ & $3.14 \pm 0.03(7.2)$ & $\ldots$ & $\ldots$ & 4.0 & 164 & 7 \\
\hline $061021 \ldots \ldots$ & \multicolumn{6}{|c|}{ Weighted Average over Four Longest Nights } & 11 \\
\hline$\ldots \ldots \ldots$ & $2.73 \pm 0.03$ & $3.12 \pm 0.02$ & $\begin{array}{c}3.53 \pm 0.03 \\
\text { mbined Data Set (See }\end{array}$ & $4.45 \pm 0.05$ & $\cdots$ & $\cdots$ & $\cdots$ \\
\hline$\ldots \ldots \ldots$ & $2.751190 \pm 0.000010$ & $3.116709 \pm 0.000006$ & $3.495113 \pm 0.000009$ & $4.443120 \pm 0.000012$ & $\ldots$ & $\ldots$ & $\ldots$ \\
\hline
\end{tabular}

NOTE.--Missing data indicates the frequency was not observed to the required significance level of $90 \%$.

${ }^{a}$ Includes only data used in analysis; excludes contaminated frames (i.e., cosmic ray in program star, clouds, poor seeing, etc.).

${ }^{\mathrm{b}}$ See $\S 4$ for discussion on the inclusion and exclusion criteria for comparison stars. There was a maximum 21 comparison stars possible. 


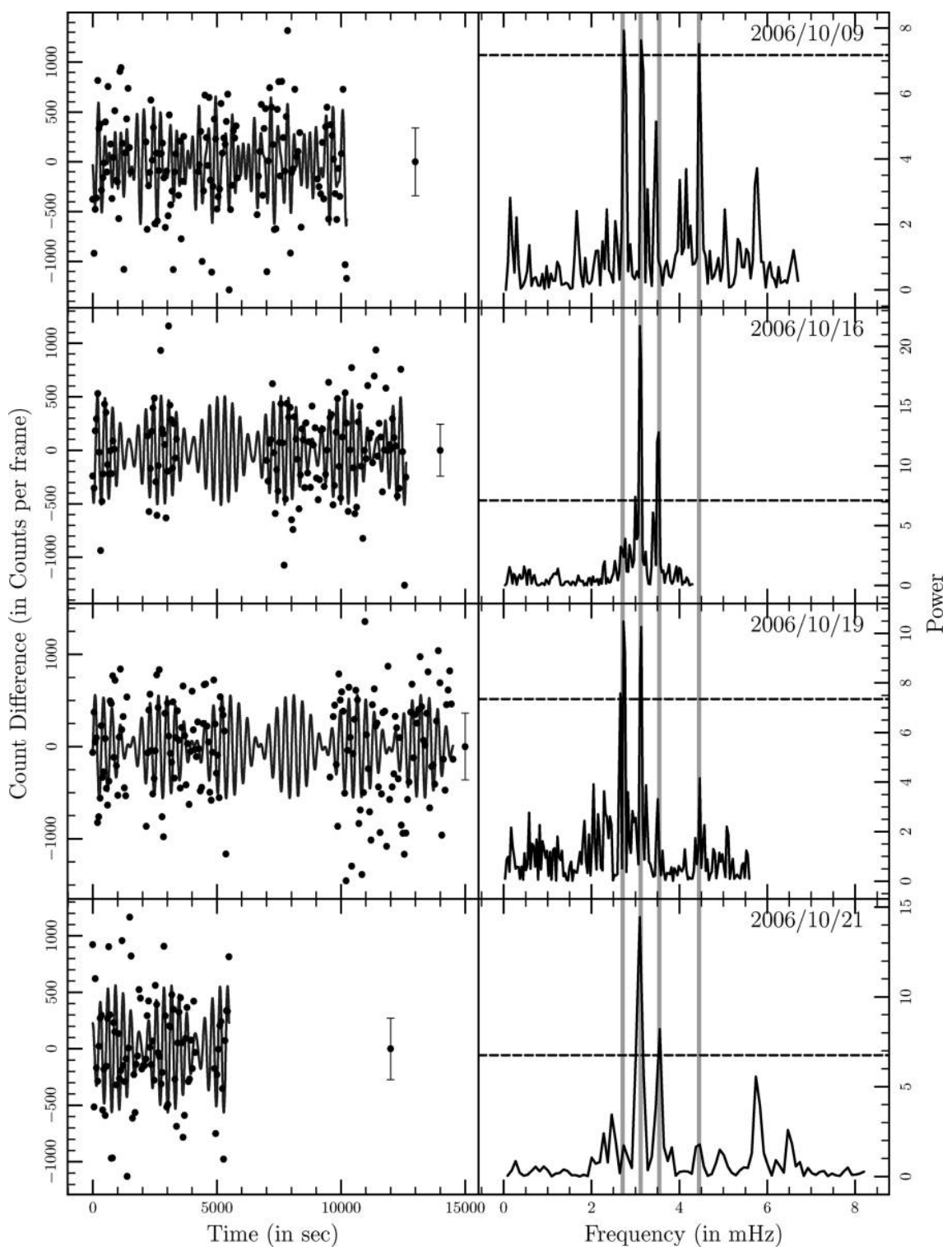

Fig. 2.-Left Panels: Light curves (see § 2.2) plotted as count difference compared to mean counts of the program star on that night vs. time. Counts refers to the total counts accumulated within the $30 \mathrm{~s}$ exposure. A least-squares fit of sinusoidal functions to the observed significant frequencies is also plotted. Points detached from data sets denote typical error bars. Right Panels: Lomb-Scargle Periodograms. (see § 3) Dashed lines denote power level required for $90 \%$ significance. Gray vertical lines show locations of $2.7,3.1,3.5$, and $4.4 \mathrm{mHz}$ frequencies.

of the fitting model requires the use of the LevenbergMarquardt method, which is given the accurate determinations of the frequencies, amplitudes, and phases of the four detected frequencies as a starting point. This method incorporates the calculation of the covariance matrix, which in turn gives us a measure of the precision of each parameter of the best-fit model. This yielded more precise values of $2.751190 \pm 0.000010$, $3.116709 \pm 0.000006,3.495113 \pm 0.000009$, and $4.443120 \pm$ $0.000012 \mathrm{mHz}$, more than a 1000 -fold increase in precision.

This new fitted sinusoidal function was then subtracted from the data and its periodogram can be found in the lower panel of Figure 3. The striking features of this designaled periodogram is the remainder of two signals of significant power near 4.44 and $5.75 \mathrm{mHz}$. However, the false-alarm probability arguments are not valid in a data set where signals have been removed artificially. Interestingly, the excess power near $4.44 \mathrm{mHz}$ is in a frequency bin significantly offset from our reported detected frequency. If we treat both of these left over frequencies as real pulsation frequencies and use our algorithm, we find that there may exist two more detected frequencies at $4.450643 \pm 0.000017$ and $5.755451 \pm 0.000018 \mathrm{mHz}$. The existence of these frequencies is questionable because neither 


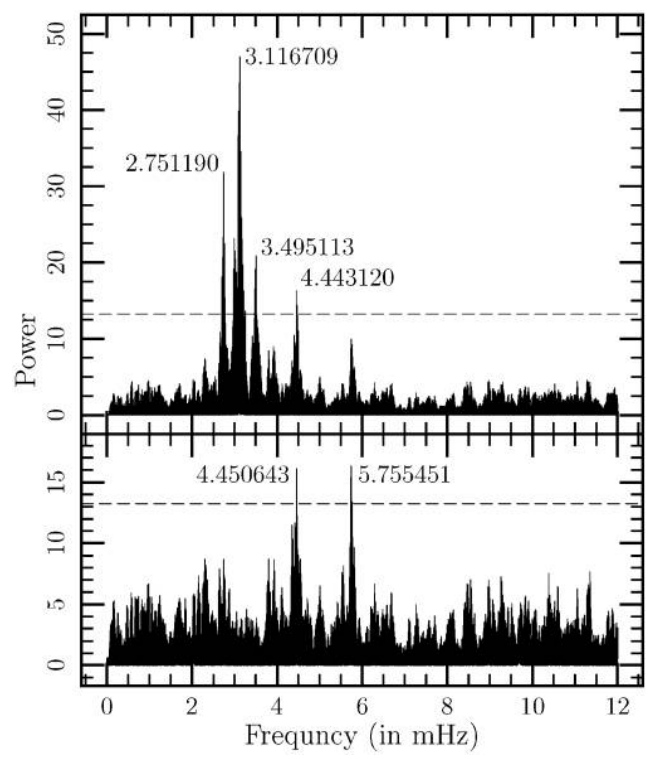

FIG. 3.-Top Panel: Lomb-Scargle Periodogram for combined data set (see $\S 4)$. Bottom Panel: Lomb-Scargle Periodogram for designaled (using only the four significant to $90 \%$ detected frequencies) data set. For both plots the dashed lines denote power level required for $90 \%$ significance.

frequency had enough power to reach our required false-alarm significance level in the full data set. Additionally, the new $4.450643 \mathrm{mHz}$ frequency is entirely lost within the window function around the original detected frequency. When we designal the entire data set with the least-squares fitted sinusoidal function including the six frequencies, the resulting periodogram no longer contains any frequency bins with significant power. The existence of these two pulsation frequencies is uncertain until better data with higher frequency sampling and more amicable window function can be obtained.

\section{CONCLUSIONS}

We have successfully detected four pulsation frequencies (periods), $2.751190 \mathrm{mHz}(363.479 \mathrm{~s}), 3.116709 \mathrm{mHz}(320.851 \mathrm{~s})$, $3.495113 \mathrm{mHz}(286.114 \mathrm{~s})$, and $4.443120 \mathrm{mHz}(225.067 \mathrm{~s})$, in multiple observations of HS $1824+6000$. There are also two possible pulsation frequencies (periods) at $4.450643 \mathrm{mHz}$ (224.687 s) and $5.755451 \mathrm{mHz}(173.748 \mathrm{~s})$. With these periods of pulsation in HS 1824, the question remains if it, or other low gravity systems, can be empirically distinguished from the normal C/O-core ZZ Ceti population. To answer this we compiled all known ZZ Ceti stars with published pulsation periods and spectroscopically measured gravities of $\log g<8.0$. This search resulted in 30 systems including HS 1824. In Figure 4 we plot all reported periods for these 30 systems. Across all of these ZZ Ceti systems there exist many reported pulsation periods ranging from 100-1400 s. However, it is apparent that better than half of the reported periods reside within the range of 150-400 s.
The four periods of HS 1824 are indistinguishable from the rest of this set of ZZ Ceti stars. Further, there does not appear to be any distinction between the two low-mass $(\log g \lesssim 7.67)$ systems (HE 0031-5525, SDSS J2135-0743; Castanheira et al. 2006) and the rest of the set. With this current set of data it appears that this empirical analysis of reported pulsation periods is not sufficient to distinguish a suspected $\mathrm{He}$ core from a normal $\mathrm{C} / \mathrm{O}$ core. However, HE 0031-5525, and SDSS J2135-0743 (Castanheira et al. 2006) are very close to the boundary of He- and C/O-core WDs and within the errors of their $\log g$ measurements may be $\mathrm{C} / \mathrm{O}$ cores.

It remains uncertain as to what degree this period spectrum comparative analysis can succeed. There are two primary differences between He- and C/O-core WDs that affect $g$-modes: the contrast in mean molecular weights in their cores, and the one fewer stratified layer in a He-core object. $g$-modes penetrate deeply into the core, so that differences in the Brunt-Väisälä profile there (due to the mean molecular weight; see Deloye $\&$ Bildsten 2002) significantly change the resulting mode period spectrum (Arras et al. 2006). The stratified layers of material within the WD also affect how different pulsation modes are trapped, driven, and excited (Córsico \& Benvenuto 2002; Arras et al. 2006). He-core WDs possess only two zones of $\mathrm{He}$ and $\mathrm{H}$, while $\mathrm{C} / \mathrm{O}$-core WDs possess the additional zone of C/O. Qualitatively, both of these differences would produce differences in the mode period spectra and are the subject of current theoretical work we are pursuing. Once these full mode calculations are available, we can answer whether clear differences are observable.

Most reported systems in Figure 4 were found in observational campaigns looking only for pulsations in an effort to constrain the ZZ Ceti instability strip. In most cases, no attempt was made to distinguish observed pulsation periods as independent modes, as opposed to linear combinations of modes. This analysis was neglected in large part due to the lack of extensive follow up. Our observations of HS 1824 showed most single nights of data contain the pulsations of one specific period and it was a rarity to find a night of data with multiple pulsation periods. Ideally, very long gapless observations on the order of several days would address these problems very well. These observations could be obtained through the use of telescope networks such as the Whole Earth Telescope ${ }^{2}$ as was done with HL Tau 76 (Dolez et al. 2006) and G117-B15A (Kepler et al. 1991, 1995) and the Las Cumbres Observatory Global Telescope. ${ }^{3}$ We look toward future, more detailed observations of many low-mass and normal-mass ZZ Cetis to help provide a measurable distinction between He- and C/O-core compositions in WDs.

\footnotetext{
${ }^{2}$ Information on the Whole Earth Telescope can be found online at http://www .physics.udel.edu/darc/wet.

${ }^{3}$ Information on the Las Cumbres Observatory Global Telescope is available online at http://www.lcogt.net.
} 


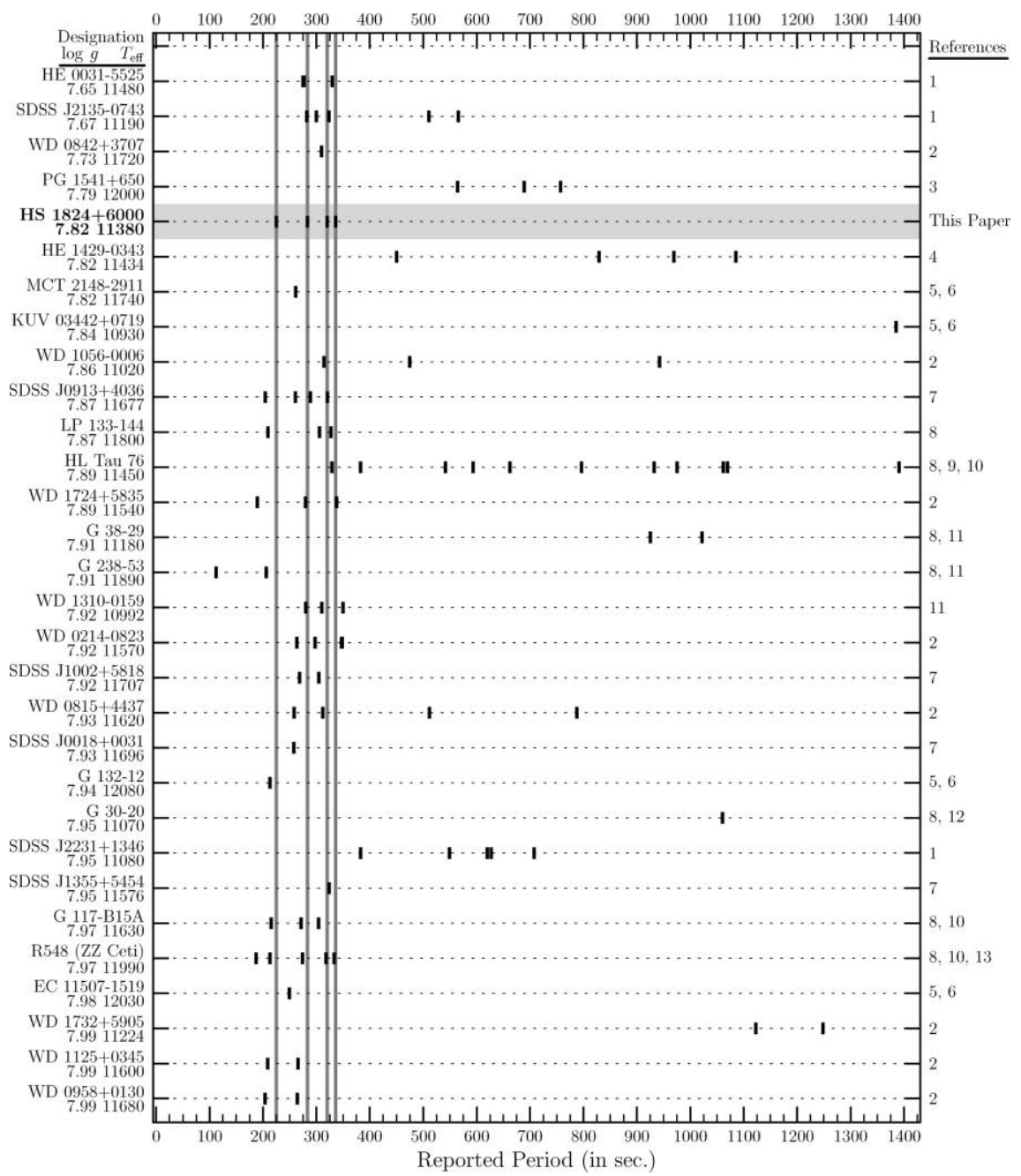

FIG. 4.-Spectrum of reported pulsation periods for published ZZ Ceti systems with spectroscopically determined $\log g<8$. HS 1824 is highlighted in gray with its four observed period locations marked with four vertical lines. Some marks represent more than one (very closely spaced) observed pulsation period; see references for details. HL Tau 76 lists only the verified independent pulsation modes of Dolez et al. (2006). Several systems listed here are not included in Fig. 1 as their $\log g$ and $T_{\text {eff }}$ measurements are not of sufficient precision. References-(1) Castanheira et al. 2006; (2) Mukadam et al. 2004a; (3) Vauclair et al. 2000; (4) Silvotti et al. 2005; (5) Gianninas et al. 2007; (6) Gianninas et al. 2006; (7) Mullally et al. 2005; (8) Bergeron et al. 2004; (9) Dolez et al. 2006; (10) Bergeron et al. 1995; (11) Kepler et al. 2005b; (12) Mukadam et al. 2002; (13) Mukadam et al. 2003.

We thank Anjum Mukadam for alerting us to the existence of this object and the referee for comments that clarified our presentation. We thank Phil Arras for useful discussion on pul- sations in He-core WDs. This work was supported by the National Science Foundation under grants PHY 05-51164 and AST 07-07633.

\section{REFERENCES}

Althaus, L. G., \& Benvenuto, O. G. 1997, ApJ, 477, 313

Althaus, L. G., Serenelli, A. M., \& Benvenuto, O. G. 2001, MNRAS, 323, 471

Arras, P., Townsley, D. M., \& Bildsten, L. 2006, ApJ, 643, L119

Bedin, L. R., Salaris, M., Piotto, G., King, I. R., Anderson, J., Cassisi, S., \& Momany, Y. 2005, ApJ, 624, L45

Bedin, L. R., King, I. R., Anderson, J., Piotto, G., Salaris, M., Cassisi, S., \& Serenelli, A. 2008a, ApJ, 678, 1279
Bedin, L. R., Salaris, M., Piotto, G., Cassisi, S., Milone, A. P., Anderson, J., \& King, I. R. 2008b, ApJ, 679, L29

Bergeron, P., Fontaine, G., Billéres, M., Boudreault, S., \& Green, E. M. 2004, ApJ, 600, 404

Bergeron, P., Wesemael, F., Lamoutague, R., Fontaine, G., Saffer, R. A., \& Allard, N. F. 1995, ApJ, 449, 258

Brassard, P., \& Fontaine, G. 1997, in White Dwarfs, ed.J. Isern, M. Hernanz, \& E. Gracia-Berro (Dordrecht: Kluwer), 451 
Castanheira, B. G., \& Kepler, S. O. 2008, MNRAS, 385, 430

Castanheira, B. G., et al. 2006, A\&A, 450, 227 2007, A\&A, 462, 989

Cenko, S. B., et al. 2006, PASP, 118, 1396

Córsico, A. H., \& Benvenuto, O. G. 2002, Ap\&SS, 279, 281

Córsico, A. H., García-Berro, E., Althaus, L. G., \& Isern, J. 2004, A\&A, 427, 923

D’Cruz, N. L., Dorman, B., Rood, R. T., \& O’Connell, R. W. 1996, ApJ, 466, 359

Deeg, H. J., \& Doyle, L. R. 2000, in Third Workshop on Photometry, ed. W. Borucki, \& L. E. Lasher, NASA/CP-2000-209614, 85

Deloy, C. J., \& Bildsten, L. 2002, ApJ, 580, 1077

Dolez, N., et al. 2006, A\&A, 446, 237

Dominguez, I., Chieffi, A., Limongi, M., \& Straniero, O. 1999, ApJ, 524, 226

Fontaine, G., Brassard, P., \& Charpinet, S. 2003, Ap\&SS, 284, 257

Fontaine, G., McGraw, J. T., Dearborn, D. S. P., Gustafson, J., \& Lacombe, P. 1982, ApJ, 258, 651

Gianninas, A., Bergeron, P., \& Fontaine, G. 2005, ApJ, 631, 1100

- 2006, AJ, 132, 831

2007, in ASP Conf. Ser. 372, 15th European Workshop on White Dwarfs, ed. R. Napiwotzki, \& M. R. Burleigh (San Francisco: ASP), 577

Gilliland, R. L., \& Brown, T. M. 1988, PASP, 100, 754

Hansen, B. M. S. 2005, ApJ, 635, 522

Horne, J. H., \& Baliunas, S. L. 1986, ApJ, 302, 757

Howell, S. B. 2006, Handbook of CCD Astronomy, Cambridge University Press, p. 66

Iben, I., \& Livio, M. 1993, PASP, 105, 1373

Kalirai, J. S., Bergeron, P., Hansen, B. M. S., Kelson, D. D., Reitzel, D. B., Rich, R. M., \& Richer, H. B. 2007, ApJ, 671, 748

Kawka, K., Vennes, S., Oswalt, T. D., Smith, J. A., \& Silvestri, N. M.. 2006, ApJ, 643, L123

Kepler, S. O., Castanheira, B. G., Saraiva, M. F. O., Nitta, A., Kleinman, S. J., Mullally, F., Winget, D. E., \& Eisenstein, D. J. 2005, A\&A, 442, 629

Kepler, S. O., Mukadam, A., Winget, D. E., Nather, R. E., Metcalfe, T. S., Reed, M. D., Kawaler, S. D., \& Bradley, P. A. 2000, ApJ, 534, L185

Kepler, S. O., et al. 1991, ApJ, 378, L45

1995, Baltic Astron., 4, 221

2005a, ApJ, 634, 1311

Kilic, M., Prieto, C. A., Brown, W. R., \& Koester, D. 2007, ApJ, 660,1451
Kilic, M., Stanek, K. Z., \& Pinsonneault, M. H. 2007, ApJ, 671, 761

Marsh, T. R., Dhillon, V. S., \& Duck, S. R. 1995, MNRAS, 275, 828

Mukadam, A. S., Kepler, S. O., Winget, D. E., \& Bergeron, P. 2002, ApJ, 580, 429

Mukadam, A. S., Winget, D. E., von Hippel, T., Montgomery, M. H., Kepler, S. O., \& Costa, A. F. M. 2005, in ASP Conf. Ser. 334, 14th European Workshop on White Dwarfs, ed. D. Koester, \& S. Moehler (San Francisco: ASP), 459

Mukadam, A. S., et al. 2003, ApJ, 594, 961

- 2004a, ApJ, 607, 982

- 2004b, ApJ, 612, 1052

Mullally, F., et al. 2005, ApJ, 625, 966

Panei, J. A., Althaus, L. G., Chen, X., \& Han, Z. 2007, MNRAS, 382, 779

Pietrinferni, A., Cassisi, S., Salaris, M., \& Castelli, F. 2004, ApJ, 612, 168

Reiger, S. H. 1963, AJ, 68, 395

Robinson, E. L., Kepler, S. O., \& Nather, R. E. 1982, ApJ, 259, 219

Robinson, E. L., et al. 1995, ApJ, 438, 908

Scargle, J. D. 1982, ApJ, 263, 835

Silvotti, R., Voss, B., Bruni, I., Koester, D., Reimers, D., Napiwotzki, R., \& Homeier, D. 2005, A\&A, 443, 195

Sokoloski, J. L., Bildsten, L., \& Ho, W. C. G. 2001, MNRAS, 326, 553

VandenBerg, D. A., Bergbusch, P. A., \& Dowler, P. D. 2006, ApJS, 162,375

Vauclair, G., Dolez, N., Fu, J.-N., Homeier, D., Roques, S., Chevreton, M., \& Koester, D. 2000, A\&A, 355, 291

Voss, B., Koester, D., Østensen, R., Napiwotzki, R., Homeier, D., \& Reimers, D. 2006, A\&A, 450, 1061

- 2007, in ASP Conf. Ser. 372, 15th European Workshop on White Dwarfs, ed. R. Napiwotzki, \& M. R. Burleigh (San Francisco: ASP), 583

Wesemael, F., Bergeron, P., Fontaine, G., \& Lamontagne, R. 1991, in NATO ASI Ser. C, 336, Proc. 7th European Workshop on White Dwarfs, ed. G. Vauclair, \& E. Sion (Dordrecht: Kluwer), 159

Wu, Y., \& Goldreich, P. 1999, ApJ, 519, 783

Young, A. T. 1967, AJ, 72, 747

Note added in proof.-The authors point out an excellent paper by L. G. Althaus, et al. 2004, MNRAS, 347, 125 of a comparative theoretical pulsation analysis of He-core and $\mathrm{O}$ core WDs concluding that observable differences in the mode period spectrum may exist. 